\title{
Knockout of Sphingosine Kinase 1 Attenuates Renal Fibrosis in Unilateral Ureteral Obstruction Model
}

\author{
Xiwen Zhang ${ }^{\text {a }}$ Weili Wang ${ }^{\text {a }}$ Xin-Ying Ji ${ }^{b}$ Joseph K. Ritter ${ }^{a}$ Ningjun Li $^{\mathrm{a}}$ \\ ${ }^{a}$ Department of Pharmacology and Toxicology, Virginia Commonwealth University School of Medicine, Richmond, \\ VA, USA; ${ }^{b}$ Henan International Joint Laboratory for Nuclear Protein Regulation, School of Basic Medical Sciences, \\ Henan University College of Medicine, Kaifeng, China
}

\section{Keywords}

Sphingosine-1-phosphate $\cdot$ a-Smooth muscle actin .

Collagen

\begin{abstract}
Background: Sphingosine-1-phosphate (S1P) is a bioactive sphingolipid metabolite involved in various diseases. S1P also plays significant roles in the differentiation of fibroblasts into myofibroblasts, being implicated in fibrotic diseases. S1P is produced by the phosphorylation of sphingosine catalyzed by sphingosine kinases (SphK1 and SphK2). It remains unclear if the activation of endogenous SphK1 contributes to fibrogenesis in kidneys. The present study determined the effect of SphK1 gene knockout (KO) on fibrotic markers in kidneys. Methods: The renal fibrosis was produced using the unilateral ureteral obstruction (UUO) model in wild-type (WT) and SphK1 gene KO mice. Renal mRNA levels of SphK1 and S1P receptors (S1PR) were measured by real-time RT-PCR. Fibrotic and immune cell markers in kidneys were measured by Western blot analysis and immunostaining, respectively. Renal morphological damage was examined by Periodic-Acid Schiff staining. $\boldsymbol{R e}$ sults: The mRNA levels of SphK1 and S1PRs were dramatically increased in renal tissues of WT-UUO mice, whereas the increase in renal SphK1 mRNA was blocked in KO-UUO mice. In-
\end{abstract}

terestingly, the increased levels of fibrotic markers, collagen and a-smooth muscle actin, in kidneys were significantly attenuated in KO-UUO versus WT-UUO mice. Meanwhile, kidney damage indices were remarkably attenuated in KO-UUO mice compared with WT-UUO mice. However, increased numbers of CD43+ and CD48+ cells, markers for $T$ cell and macrophage, respectively, showed no significant difference between WT-UUO and KO-UUO kidneys. Conclusion: The activation of the SphK1-S1P pathway may contribute to tubulointerstitial fibrosis in UUO kidneys by affecting fibrotic signaling within renal cells independent of immune modulation.

(C) 2019 S. Karger AG, Basel

\section{Introduction}

Sphingosine-1-phosphate (S1P) is an important signaling molecule in various organ systems including the cardiovascular system and kidney [1-3]. The production of S1P is catalyzed by sphingosine kinases (SphKs), which phosphorylate sphingosine to S1P [1-3]. S1P works in both intracellular and extracellular pathways [3]. Currently, there is limited information showing that S1P catalyzed by SphK2 acts on intracellular targets and produces effects that are receptor-independent $[3,4]$.

\section{KARGER}

(c) 2019 S. Karger AG, Basel

E-Mail karger@karger.com

www.karger.com/ajn
Dr. Ningjun Li

Department of Pharmacology and Toxicology

Medical College of Virginia Campus, Virginia Commonwealth University

PO Box 980613, Richmond, VA 23298 (USA)

E-Mail nli@vcu.edu 
When acting as an extracellular signaling molecule, S1P is synthesized in the cytosol by SphK1 [4] and then exported out of the cell to target its receptors on plasma membrane [5-8]. The effects of S1P are mostly mediated by its cell surface receptors. There are $5 \mathrm{G}$-protein-coupled S1P receptors (S1PR), S1PR1, S1PR2, S1PR3, S1PR4, and S1PR5 [9]. S1PR1, S1P2, and S1PR3 are expressed in almost all the tissues, whereas S1PR4 and S1PR5 are expressed mainly in lymphoid and brain, respectively [10]. A large number of studies have shown that S1P participates in multiple kidney injuries or diseases, such as ischemia-reperfusion injury, diabetic nephropathy, glomerulonephritis, nephroblastoma, and renal fibrosis [11-14].

Studies have demonstrated that S1P signaling interacts with TGF- $\beta 1$ signaling to produce a vicious cycle in fibrosis [15]. S1P binds to S1PR2 and S1PR3 to initiate fibrogenesis by interacting with Smad complexes. Meanwhile, the expression of SphK1 is also enhanced by TGF- $\beta 1$ signaling through the activation of Smad complex, which increases the synthesis of S1P. The increased production of S1P via its receptors further amplifies the fibrogenesis process $[1,16,15]$. Although there have been reports showing that $\mathrm{S} 1 \mathrm{P}$ signaling participates in fibrogenesis in other organs and kidney tissue, there is no direct evidence showing the role of endogenous SphK1 in renal fibrosis in vivo. The present study investigated the role of SphK1 in renal fibrosis using SphK1 knockout $(\mathrm{KO})$ mice in the model of unilateral ureteral obstruction (UUO).

\section{Materials and Methods}

\section{Animals and the UUO Model}

Experiments were performed in 3 month old 25-30 g male C57BL/6 mice and SphK1 KO mice generated on the C57BL/6 background. Mice were purchased from the Jackson Laboratory and bred in our animal facility. All protocols were approved by the Institutional Animal Care and Use Committee of the Virginia Commonwealth University.

The UUO surgery was performed as described previously [17]. Mice were anesthetized with inhaled isoflurane (2.5\%). The midline longitudinal abdominal incision was performed, which permitted access to the left kidney and ureter. The left ureter was tied at the level of the lower pole of the kidney with surgical silk. Special attention was paid to ensure that the point of ligation was identical and that the damage to the kidneys would be the same in UUO kidneys across animals. All the mice underwent UUO surgery to affect the left kidney and that the unobstructed contralateral kidneys were used as control. The mice were sacrificed after 14 days and both kidneys were collected. The kidney samples were divided into 4 groups: Wild-type control (WT-C),
WT-UUO, SphK1 KO control (KO-C), SphK1 KO UUO (KO-UUO). Half of each kidney was fixed in $10 \%$ neutral buffered formalin and the other half frozen in liquid $\mathrm{N}_{2}$ and stored at $-80^{\circ} \mathrm{C}$.

\section{RNA Isolation and Real-Time RT-PCR}

Total RNA was isolated from mouse kidney tissue using TRIzol reagent (Life Technology, Rockville, MD, USA) and reverse-transcribed using the cDNA synthesis kit (Bio-Rad, Hercules, CA, USA). The reverse transcribed cDNA products were amplified using a TaqMan Gene Expression Assays kit (Applied Biosystems). A TaqMan gene expression assays kit for detecting the level of $18 \mathrm{~S}$ rRNA was used as an endogenous control. The relative SphK1 mRNA levels were calculated in accordance with the $\Delta \Delta \mathrm{Ct}$ method and expressed as $2^{-\Delta \Delta \mathrm{Ct}}$.

\section{Protein Preparation and Western Blot Analysis}

The kidney samples were prepared for Western blot analysis as we described before $[18,19]$. In brief, renal tissues were homogenized with a glass homogenator in ice-cold RIPA lysis buffer and the homogenate then centrifuged at $5,000 \mathrm{~g}$ for $5 \mathrm{~min}$ at $4{ }^{\circ} \mathrm{C}$. The supernatant was collected and subjected to Western blot analysis with antibodies against collagen I/III (rabbit 1:2000, Calbiochem), or $a$-smooth muscle actin ( $\alpha$-SMA; rabbit 1:2000, Abcam), and glyceraldehyde 3-phosphate dehydrogenase (GAPDH) (rabbit 1: 3000, Cell Signaling Technology) as loading control.

\section{Immunohistochemistry}

The renal tissue was fixed in $10 \%$ neutral buffered formalin, paraffin-embedded, and cut into 5 - $\mu$ m sections. Immunostaining was performed as we described before [20,21], using antibodies against CD43 (1:100, Santa Cruz Biotechnology) and CD68 (1:100, Santa Cruz Biotechnology). Collagen I/III was stained using Picro Sirius Red. The percentage of positive-stained area was calculated using a computer program Image-Pro Plus [22].

\section{Morphological Examinations}

Glomerular and tubular structures were examined in paraffinembedded kidney sections stained with Periodic-Acid Schiff staining (Sigma-Aldrich staining kit). Glomerular and tubular injuries were scored on a scale of $0-4$ depending on the extent of sclerotic changes. Tubular injury score and glomerular damage index were scored semiquantitatively by 2 blinded examiners on the minimum of 20 cortical fields in $\times 400$ magnification as described before [23-25]. The tubular injury is defined as tubular dilatation, tubular atrophy, sloughing of tubular epithelial cells, or thickening of the tubular basement membrane, and the glomerular damage is defined as segmental or global capillary collapse with increased matrix deposition. The score was calculated on a scale of $0-4$, as follows: $0=$ no injury; $1=1-25 \%$ injured area; $2=$ $26-50 \%$ injured area; $3=51-75 \%$ injured area; $4>75 \%$ injured area $[23-25]$.

\section{Statistical Analyses}

Data are expressed as the mean \pm SEM. Comparisons among groups were performed with two-way analysis of variance followed by Tukey's test to examine the effects of gene deletion and UUO treatment as the independent factors. $p<0.05$ was considered statistically significant. 


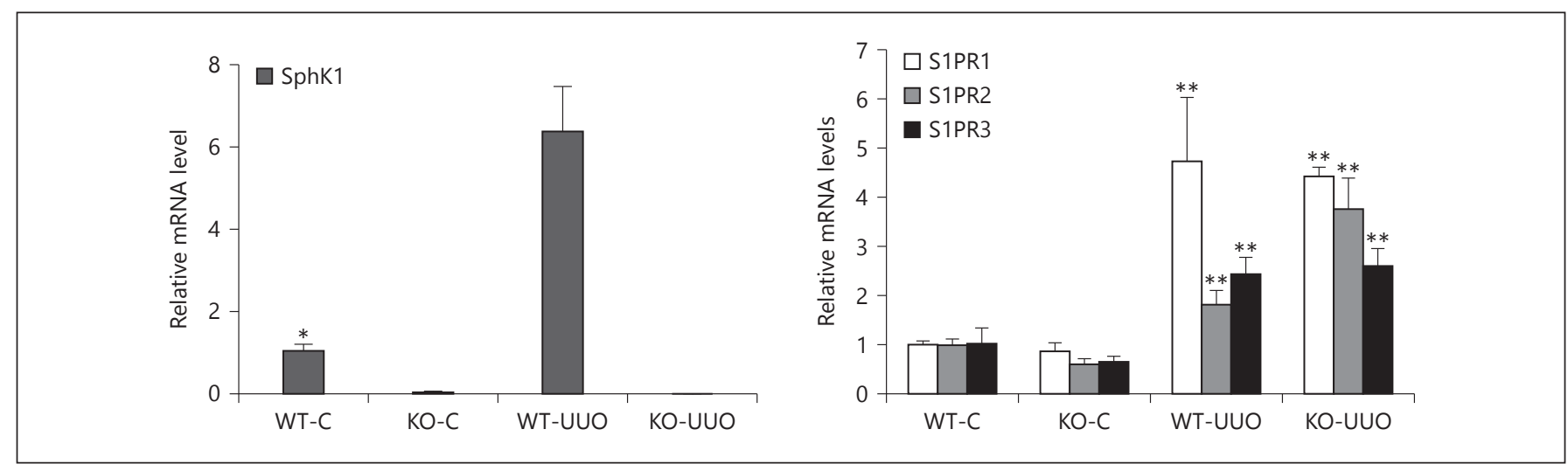

Fig. 1. Effect of UUO and SphK1 KO on mRNA levels of SphK1 and S1PR: relative mRNA levels of SphK1 and S1PRs in different groups measured by real-time RT-PCR analysis. The results were presented as fold change normalized with the WT-C. ${ }^{*} p<0.05$ versus all other groups, ${ }^{* *} p<0.05$ versus WT-C and KO-C groups
( $n=4-6)$. SphK1, sphingosine kinase 1; WT-C, wild-type control; $\mathrm{KO}-\mathrm{C}$, knockout control; WT-UUO, wild-type unilateral ureteral obstruction; KO-UUO, knockout unilateral ureteral obstruction; S1PR, S1P receptors.

\section{Results}

\section{Enhanced mRNA Expression of SphK1 and S1P Receptors in UUO Model}

Figure 1 shows the relative levels of SphK1 mRNA, which was significantly increased by 6 fold in WT-UUO compared with WT-C, whereas the SphK1 mRNA was barely detectable in either $\mathrm{KO}-\mathrm{C}$ or $\mathrm{KO}$-UUO. It is suggested that the UUO induces expression of SphK1, which is prevented in SphK1 KO mice. S1PRs 1-3 were also increased in UUO kidneys. There was a tendency toward reduced levels of S1PR mRNAs in KO-C kidneys, though it did not reach statistical significance. There was no significant difference between WT-UUO and KO-UUO kidneys. These data suggested that there are activations in S1P pathways in response to UUO.

\section{Attenuated Expression of Fibrotic Markers $\alpha$-SMA} and Collagen I in SphK1 KO Mice in the UUO Model

The levels of collagen I and $\alpha$-SMA determined by Western blot analysis (Fig. 2a) were significantly increased in both UUO groups (Fig. 2b, c). However, the levels of a-SMA and collagen I were significantly lower in KO-UUO than in WT-UUO (Fig. 2). The percentage of collagen staining area was also notably higher in UUO groups than in control groups, but was much lower in KO-UUO than in WT-UUO (Fig. 2d, e), which was consistent with the result of Western blot analysis. It is suggested that the deletion of SphK1 inhibits fibrogenesis in UUO-induced renal fibrosis.
Attenuated Morphological Injury Induced by UUO in SphK1 KO Mice

To detect whether KO of SphK1 was protective against kidney damage induced by UUO, the morphological injury was evaluated for glomerular damage index and tubular injury score based on Periodic-Acid Schiff staining. As shown in Figure 3a, glomeruli and tubules in UUO tissue showed a typical pathological damage profile. There was expanded mesangial matrix with hypercellularity, capillary collapse, and fibrous deposition in the glomeruli and tubular atrophy and vacuolar degeneration in the tubule epithelia. Summarized tubular injury scores and glomerular damage indices were shown in Figures $3 b$ and $c$. The injury scores were notably higher in WT-UUO and KO-UUO than that in WT-C and KO-C, respectively, but much lower in KO-UUO than in WT-UUO. It is suggested that $\mathrm{KO}$ of SphK1 is protective in the kidney in the UUO model.

\section{No Significant Difference in the Infiltration of}

Immune Cells between WT-UUO and KO-UUO

$\mathrm{S} 1 \mathrm{P}$ signaling is also involved in immune regulation. We wanted to determine whether the differences in renal fibrotic markers between WT-UUO and KO-UUO were due to the immune regulation by KO of SphK1 and detected the immune cell infiltration by staining CD43, a T-cell marker, and CD68, a macrophage marker. Immunohistochemical analysis showed that CD43+ and CD68+ cells were notably increased in both UUO groups compared with control groups (Fig. 4a). However, there was 


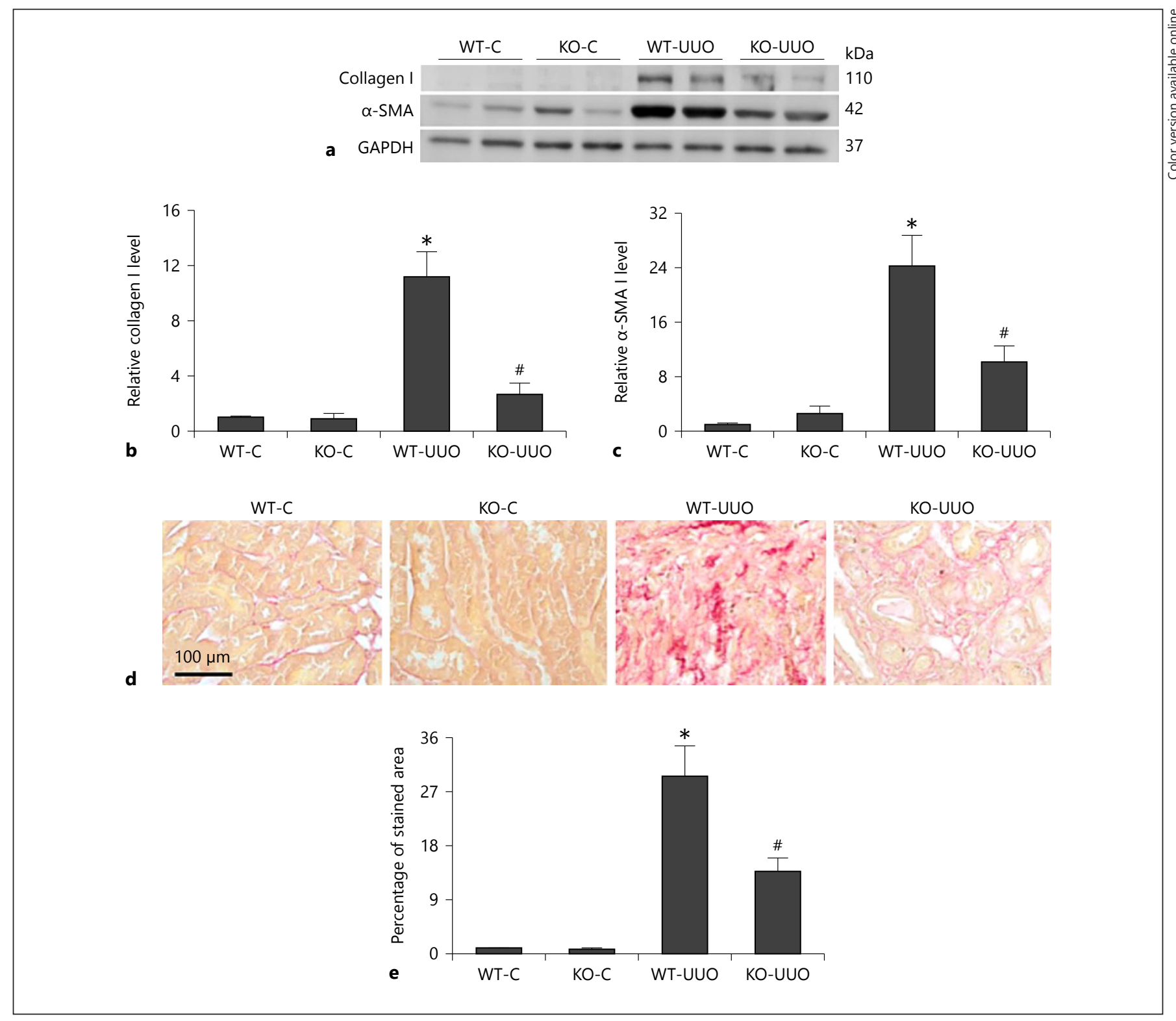

Fig. 2. Effect of UUO and SphK1 KO on the expression of fibrotic markers collagen I and SMA (a color version is available in online supplementary). a Representative Western blot gel documents showing Collagen I and $\alpha$-SMA levels. b, c Summarized data showing the levels of collagen I and $\alpha$-SMA as the ratio of band intensities versus GAPDH normalized to WT-C. d Representative photomicrographs of Sirius Red-stained kidney sections showing the

no significant difference in the positive staining of CD43 or CD68 between WT-UUO and KO-UUO (Fig. 4b, c, respectively), indicating that there is no difference in the immune cell infiltration in the UUO kidneys between KO and WT mice.

SphK1 and Renal Fibrosis positive collagen staining area in red. $\mathbf{e}$ The average percentage of collagen staining quantified using Image-Pro Plus software. ${ }^{*} \#$ $p<$ 0.05 versus all other groups $(n=5)$. WT-C, wild-type control; KO-C, knockout control; WT-UUO, wild-type unilateral ureteral obstruction; KO-UUO, knockout unilateral ureteral obstruction; a-SMA, $\alpha$-smooth muscle actin.

\section{Discussion}

The present study demonstrated that Sphk1 was dramatically upregulated in the UUO model, that the increases in levels of fibrotic markers, a-SMA and collagen, as well as kidney morphological damage scores induced 


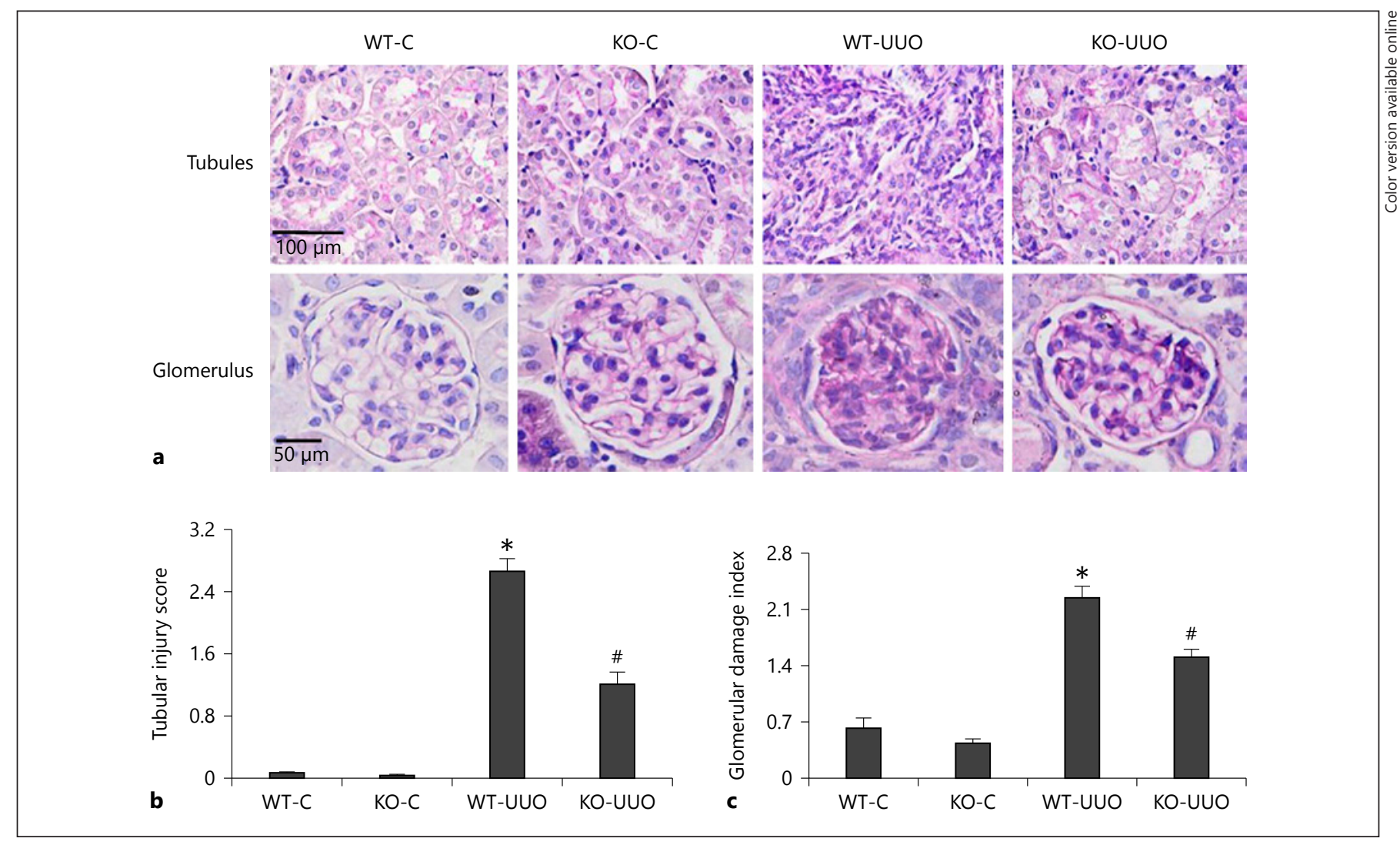

Fig. 3. Effect of UUO and SphK1 KO on morphological injury scores in kidneys (a color version is available in online supplementary). a Representative photomicrographs showing tubular and glomerular structures by Periodic-Acid Schiff staining. b, c Summarized tubular injury score and glomerular damage index by semi-quantitation. ${ }^{*}{ }^{\#} p<0.05$ versus all other groups $(n=5)$. WT-C, wild-type control; KO-C, knockout control; WT-UUO, wild-type unilateral ureteral obstruction; KO-UUO, knockout unilateral ureteral obstruction. by UUP were significantly blocked in SphK1 KO mice, and that the increases in staining for CD43, a T cell marker, and CD68, a macrophage marker, were not different between WT-UUO and KO-UUO kidneys. These results suggest that deletion of the SphK1 gene protects the kidney from damage independent of the immune regulatory action in the UUO model.

At first, we observed that the mRNA expression of SphK1 and S1PRs 1-3 was remarkably increased in the kidneys of WT-UUO group compared with WT-C group, suggesting activations of S1P signaling pathways in response to UUO. The increased level of SphK1 in this renal fibrotic model was consistent with the findings from a previous study showing that SphK1 overexpression transgenic mice produces cardiac fibrosis [26]. The increased expression of SphK1 observed in our study suggested that upregulated SphK1 might participate in the renal fibrosis in the UUO model. We then went on to examine whether deletion of SphK1 to block the increase in SphK1 protected the kidneys in this model.

Our results showed that the increase in fibrotic markers induced by UUO was considerably attenuated in SphK1 KO mice, suggesting that overproduction of S1P may be responsible for the renal fibrosis. These results are in line with the findings that $\mathrm{S} 1 \mathrm{P}$ is involved in different types of fibrosis and that the endogenous $\mathrm{S} 1 \mathrm{P}$ is increased in liver tissues in cholestasis-induced liver fibrosis [27]. Consistently, it has been shown that exogenous S1P induces fibrogenesis in NRK49F cells, a normal rat kidney fibroblast cell line, in vitro [28]. In the latter study, it was also shown that renal fibrosis, interstitial injury, and the activity of cell migration were decreased in UUO model by antagonism of S1PRs with nonselective functional antagonist FTY720 or inhibiting SphKs with DMS, a SphK inhibitor, in vivo [28]. However, we detected the effect of endogenous SphK1 using a genetic KO model, which 


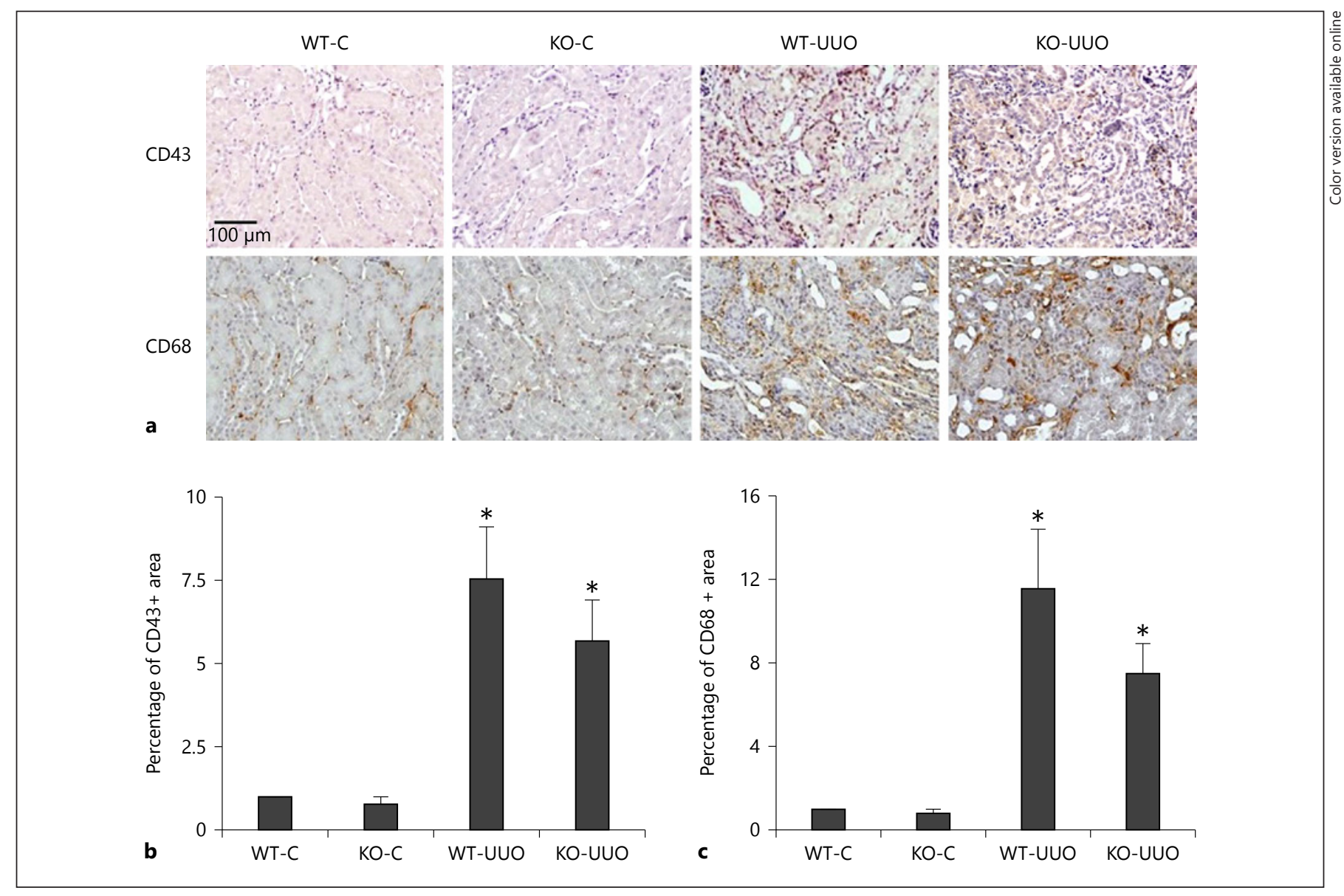

Fig. 4. Effect of UUO and SphK1 KO on the infiltration of immune cells (a color version is available in online supplementary). a Representative photomicrographs of immunohistochemistry of kidney sections stained for either CD43+ or CD68+ indicated by brown color. b, c The percentage of CD43+ and CD68+ staining area quantified by Image-Pro Plus software. ${ }^{*} p<0.05$ versus WT-C and KO-C $(n=5)$. WT-C, wild-type control; KO-C, knockout control; WT-UUO, wild-type unilateral ureteral obstruction; KO-UUO, knockout unilateral ureteral obstruction.

The tubular cell death has a close association with progressive renal interstitial fibrosis. In addition, it also has been stated that UUO affects the glomerular filtration rate with an $80 \%$ reduction, and that the number of glomerulus is reduced by $40 \%$. It can be concluded that UUO produces both tubular and glomerular injuries $[17,30]$. To determine the role of SphK1 in UUO-induced kidney morphological damage, the tubular injury score and glomerular damage index were assessed as endpoints. As expected, tubular injury and glomerular damage induced by UUO were attenuated in SphK1 KO mice. The results suggest targeting of SphK1 and downstream S1P signaling could be a new strategy to protect kidney structure and renal function from CKD.

The immune response is also involved in the development of the fibrogenesis [31]. The previous study has 
shown that the infiltration of $\mathrm{T}$ cells, which represents the adaptive immune response, and the infiltration of macrophages, which represents the innate immune response, start after 4-h-obstruction in UUO and that inflammatory cells progressively infiltrate into kidney tissue for the next 14 days [32]. S1P is known to be involved in multiple inflammatory responses such as recruitment of immune cells into injured tissue. Moreover, it is reported that S1P gradients regulate innate and adaptive immune cells trafficking through S1PRs [33]. Given the role of S1P signaling in the regulation of immune response and inflammation, we wondered if SphK1 KO could alter the inflammatory process and thereby attenuate fibrosis in UUO. Immunohistochemistry showed that in SphK1 KO-UUO, the increased infiltration of inflammatory cells was not significantly different from that in WT-UUO. These results indicate that the SphK1/S1P pathway is probably the intrinsic direct mediator of tubulointerstitial fibrosis in renal cells, rather than immune modulator in the fibrogenesis of UUO. In support of our findings, another study used nude mice that were athymic animals with deficiency of $\mathrm{T}$ cells and found that renal tubulointerstitial fibrosis and kidney injury induced by UUO in these immune deficient mice could also be significantly attenuated by inhibiting S1P signaling [34]. These reports together with our findings in the present study lead to a conclusion that SphK1/S1P acts on renal cells to produce renal injuries in CKD by bypassing the regulation of immune response.

Our study was not intended to explore the mechanisms by which SphK1 was increased in UUO kidneys. In this regard, the interaction between TGF- $\beta 1 /$ Smad and SphK1 pathways could be a potential mechanism to activate SphK1 [15]. It has been shown that the SphK1/S1P/ S1PRs pathways mediate TGF- $\beta 1$-induced fibrotic effects, as a S1PR3 antagonist attenuated the fibrosis in the UUO model and inhibited TGF- $\beta 1$ [35]. On the other hand, the SphK1 pathway may also be a downstream me- diator of TGF- $\beta 1$. Two different studies showed that cell transdifferentiation into myofibroblast induced by TGF- $\beta 1$ was dependent on SphK1 and S1PR3 $[36,37]$. TGF- $\beta 1$ may activate SphK1 gene transcription through the Smad complex. Thus, there may be complex interactions between the SphK1 and TGF- $\beta 1$ signaling pathways that cause increase in SphK1 in UUO kidneys. In addition, the present study was performed in UUO model. As S1P pathway has been implicated in fibrogenesis in other organs [38-40] as well as other types of CKD, such as diabetic nephropathy [41], it is very possible that activation of SphK1 pathway is also involved in other types of $\mathrm{CKD}$, which requires further investigation.

In summary, the present study revealed that deletion of SphK1 attenuated the renal tubulointerstitial fibrosis and kidney damage without significant effect on the infiltration of inflammatory cells in UUO model. These results suggest that activation of S1P production may be a new triggering mechanism of renal fibrosis. These results may provide new therapeutic strategies associated with manipulating SphK1/S1P pathway in the management of chronic kidney diseases.

\section{Disclosure Statement}

The authors have no conflicts of interest to declare.

\section{Funding Sources}

National Institutes of Health (HL145163, DK107991, and DK102539).

\section{Author Contributions}

X.Z. and W.W.: performed experiments; X.Z., J.K.R., and N.L.: analyzed data; X.Z., X.-Y.J., J.K.R., and N.L.: prepared concept and manuscript.

\section{References}

1 Jo SK, Bajwa A, Awad AS, Lynch KR, Okusa MD. Sphingosine-1-phosphate receptors: biology and therapeutic potential in kidney disease. Kidney Int. 2008 Jun;73(11):1220-30.

2 Peters SL, Alewijnse AE. Sphingosine1-phosphate signaling in the cardiovascular system. Curr Opin Pharmacol. 2007 Apr; 7(2): 186-92.

3 Maceyka M, Harikumar KB, Milstien S, Spiegel S. Sphingosine-1-phosphate signaling and its role in disease. Trends Cell Biol. 2012 Jan; 22(1):50-60.

4 Maceyka M, Sankala H, Hait NC, Le Stunff H, Liu H, Toman R, et al. SphK1 and SphK2, sphingosine kinase isoenzymes with opposing functions in sphingolipid metabolism. J Biol Chem. 2005 Nov;280(44):3711829.

5 Tamama K, Okajima F. Sphingosine 1-phosphate signaling in atherosclerosis and vascu- lar biology. Curr Opin Lipidol. 2002 Oct; 13(5):489-95.

6 Liu H, Zhang Z, Li P, Yuan X, Zheng J, Liu J, et al. Regulation of S1P receptors and sphingosine kinases expression in acute pulmonary endothelial cell injury. PeerJ. 2016 Dec;4:e2712.

7 Pyne NJ, Tonelli F, Lim KG, Long JS, Edwards J, Pyne S. Sphingosine 1-phosphate signalling in cancer. Biochem Soc Trans. 2012 Feb;40(1): 94-100. 
8 Maceyka M, Spiegel S. Sphingolipid metabolites in inflammatory disease. Nature. 2014 Jun;510(7503):58-67.

9 Nagahashi M, Yamada A, Aoyagi T, Terracina KP, Koyama Y, Wakai T, et al. Sphingosine1-Phosphate Signaling Targeted Therapy Suppresses Obesity-Related Breast Cancer Progression and Improves Survival. J Am Coll Surg. 2014;219(3):S126.

10 Hisano Y, Nishi T, Kawahara A. The functional roles of S1P in immunity. J Biochem. 2012 Oct;152(4):305-11.

11 Geoffroy K, Troncy L, Wiernsperger N, Lagarde M, El Bawab S. Glomerular proliferation during early stages of diabetic nephropathy is associated with local increase of sphingosine-1-phosphate levels. FEBS Lett. 2005 Feb;579(5):1249-54.

12 Lai LW, Yong KC, Igarashi S, Lien YH. A sphingosine-1-phosphate type 1 receptor agonist inhibits the early $\mathrm{T}$-cell transient following renal ischemia-reperfusion injury. Kidney Int. 2007 Jun;71(12):1223-31.

13 Awad AS, Rouse MD, Khutsishvili K, Huang L, Bolton WK, Lynch KR, et al. Chronic sphingosine 1-phosphate 1 receptor activation attenuates early-stage diabetic nephropathy independent of lymphocytes. Kidney Int. 2011 May;79(10):1090-8.

14 Park SW, Kim M, Kim M, D’Agati VD, Lee HT. Sphingosine kinase 1 protects against renal ischemia-reperfusion injury in mice by sphingosine-1-phosphate1 receptor activation. Kidney Int. 2011 Dec;80(12):131527.

15 Zhang X, Ritter JK, Li N. Sphingosine-1-phosphate pathway in renal fibrosis. Am J Physiol Renal Physiol. 2018 Oct;315(4):F752-6.

16 Koch A, Pfeilschifter J, Huwiler A. Sphingosine 1-phosphate in renal diseases. Cell Physiol Biochem. 2013;31(6):745-60.

17 Chevalier RL, Forbes MS, Thornhill BA. Ureteral obstruction as a model of renal interstitial fibrosis and obstructive nephropathy. Kidney Int. 2009 Jun;75(11):1145-52.

18 Zhu Q, Wang Z, Xia M, Li PL, Zhang F, Li N. Overexpression of HIF-1 1 a transgene in the renal medulla attenuated salt sensitive hypertension in Dahl S rats. Biochim Biophys Acta. 2012 Jun;1822(6):936-41.

19 Zhu Q, Liu M, Han WQ, Li PL, Wang Z, Li N Overexpression of HIF prolyl-hydoxylase-2 transgene in the renal medulla induced a salt sensitive hypertension. J Cell Mol Med. 2012 Nov; 16(11):2701-7.

$20 \mathrm{Hu}$ J, Zhu Q, Xia M, Guo TL, Wang Z, Li PL, et al. Transplantation of mesenchymal stem cells into the renal medulla attenuated saltsensitive hypertension in Dahl S rat. J Mol Med (Berl). 2014 Nov;92(11):1139-45.

21 Zhu Q, Li XX, Wang W, Hu J, Li PL, Conley $S$, et al. Mesenchymal stem cell transplantation inhibited high salt-induced activation of the NLRP3 inflammasome in the renal medulla in Dahl S rats. Am J Physiol Renal Physiol. 2016 Apr;310(7):F621-7.

22 Turnberg D, Lewis M, Moss J, Xu Y, Botto M, Cook HT. Complement activation contributes to both glomerular and tubulointerstitial damage in adriamycin nephropathy in mice. J Immunol. 2006 Sep;177(6):4094-102.

23 Wang Z, Zhu Q, Li PL, Dhaduk R, Zhang F, Gehr TW, et al. Silencing of hypoxia-inducible factor-1a gene attenuates chronic ischemic renal injury in two-kidney, one-clip rats. Am J Physiol Renal Physiol. 2014 May; 306(10):F1236-42.

24 Thallas-Bonke V, Jha JC, Gray SP, Barit D, Haller H, Schmidt HH, et al. Nox-4 deletion reduces oxidative stress and injury by PKC- $\alpha-$ associated mechanisms in diabetic nephropathy. Physiol Rep. 2014 Nov;2(11):2.

25 Komada T, Usui F, Kawashima A, Kimura H, Karasawa T, Inoue Y, et al. Role of NLRP3 Inflammasomes for Rhabdomyolysis-induced Acute Kidney Injury. Sci Rep. 2015 Jun;5(1): 10901.

26 Takuwa N, Ohkura S, Takashima S, Ohtani $\mathrm{K}$, Okamoto Y, Tanaka T, et al. S1P3-mediated cardiac fibrosis in sphingosine kinase 1 transgenic mice involves reactive oxygen species. Cardiovasc Res. 2010 Feb;85(3): 484-93.

27 Li C, Jiang X, Yang L, Liu X, Yue S, Li L. Involvement of sphingosine 1-phosphate (SIP)/ S1P3 signaling in cholestasis-induced liver fibrosis. Am J Pathol.2009 Oct;175(4):1464-72.

28 Shiohira S, Yoshida T, Sugiura H, Nishida M, Nitta K, Tsuchiya K. Sphingosine-1-phosphate acts as a key molecule in the direct mediation of renal fibrosis. Physiol Rep. 2013 Dec;1(7):e00172.

29 Han WQ, Zhu Q, Hu J, Li PL, Zhang F, Li N. Hypoxia-inducible factor prolyl-hydroxylase-2 mediates transforming growth factor beta 1-induced epithelial-mesenchymal transition in renal tubular cells. Biochim Biophys Acta. 2013 Jun;1833(6):1454-62.

30 Chevalier RL, Thornhill BA, Chang AY. Unilateral ureteral obstruction in neonatal rats leads to renal insufficiency in adulthood. Kidney Int. 2000 Nov;58(5):1987-95.

31 Sakai T, Kawamura T, Shirasawa T. Mizoribine improves renal tubulointerstitial fibrosis in unilateral ureteral obstruction (UUO)treated rat by inhibiting the infiltration of macrophages and the expression of alphasmooth muscle actin. J Urol. 1997 Dec;158(6): 2316-22.

32 Schreiner GF, Harris KP, Purkerson ML, Klahr S. Immunological aspects of acute ureteral obstruction: immune cell infiltrate in the kidney. Kidney Int. 1988 Oct;34(4):487-93.

33 Chi H. Sphingosine-1-phosphate and immune regulation: trafficking and beyond. Trends Pharmacol Sci. 2011 Jan;32(1):1624.

34 Yoo KH, Thornhill BA, Forbes MS, Coleman CM, Marcinko ES, Liaw L, et al. Osteopontin regulates renal apoptosis and interstitial fibrosis in neonatal chronic unilateral ureteral obstruction. Kidney Int. 2006 Nov;70(10): 1735-41.

35 Liu N, Tolbert E, Pang M, Ponnusamy M, Yan $\mathrm{H}$, Zhuang S. Suramin inhibits renal fibrosis in chronic kidney disease. J Am Soc Nephrol. 2011 Jun;22(6):1064-75.

36 Yang L, Chang N, Liu X, Han Z, Zhu T, Li C, et al. Bone marrow-derived mesenchymal stem cells differentiate to hepatic myofibroblasts by transforming growth factor- $\beta 1$ via sphingosine kinase/sphingosine 1-phosphate (S1P)/S1P receptor axis. Am J Pathol. 2012 Jul;181(1):85-97.

37 Cencetti F, Bernacchioni C, Nincheri P, Donati $C$, Bruni $P$. Transforming growth factorbeta1 induces transdifferentiation of myoblasts into myofibroblasts via up-regulation of sphingosine kinase-1/S1P3 axis. Mol Biol Cell. 2010 Mar;21(6):1111-24.

38 Means CK, Brown JH. Sphingosine-1-phosphate receptor signalling in the heart. Cardiovasc Res. 2009 May;82(2):193-200.

39 Li CY, Kong YX, Wang H, Wang SL, Yu H, Liu $X$, et al. Homing of bone marrow mesenchymal stem cells mediated by sphingosine 1-phosphate contributes to liver fibrosis. J Hepatol. 2009 Jun;50(6):1174-83.

40 Kono Y, Nishiuma T, Nishimura Y, Kotani $Y$, Okada T, Nakamura S, et al. Sphingosine kinase 1 regulates differentiation of human and mouse lung fibroblasts mediated by TGF-beta1. Am J Respir Cell Mol Biol. 2007 Oct; 37(4):395-404.

41 Ishizawa S, Takahashi-Fujigasaki J, Kanazawa Y, Matoba K, Kawanami D, Yokota T, et al. Sphingosine-1-phosphate induces differentiation of cultured renal tubular epithelial cells under Rho kinase activation via the S1P2 receptor. Clin Exp Nephrol. 2014 Dec;18(6): $844-52$. 